\title{
Computed tomography in the evaluation of the anomalous origin of the coronary artery: coexistence with other congenital heart disease in an adult population
}

\author{
M. Krupiński ${ }^{1}$, M. Urbańczyk-Zawadzka ${ }^{1}$, B. Laskowicz ${ }^{1}$, M. Irzyk ${ }^{1}$, R. Banyś ${ }^{1}$, \\ K. Gruszczyńska², J. Baron² \\ ${ }^{1}$ Department of Radiology and Diagnostic Imaging, John Paul II Hospital, Krakow, Poland \\ ${ }^{2}$ Department of Radiology and Nuclear Medicine, Medical University of Silesia, Katowice, Poland \\ [Received 9 April 2014; Accepted 23 July 2014]
}

Background: The aim of the study is to assess the prevalence of anomalous origin of the coronary artery in cardiac computed tomography (CT) and to evaluate its coexistence with other congenital heart disease.

Materials and methods: 7,115 patients, who underwent 64-slice or dual source cardiac CT in the years 2005-2011 were screened for the presence of anomalous origin of the coronary artery.

Results: Anomalous origin of coronary artery was found in 62 (0.87\%) patients (34 males, mean age $57.4 \pm 15.1$ years). Anomalous aortic and pulmonary origin of coronary artery concerned $59(0.83 \%)$ and $3(0.04 \%)$ cases, respectively. Concomitant heart defects were observed in $5(0.07 \%)$ patients, all with anomalous aortic origin of coronary artery. Malformations included transposition of great arteries (4 patients) and single ventricle in (1 patient).

Conclusions: The incidence of anomalous origin of a coronary artery in cardiac $C T$ is similar to invasive coronary angiography. In an adult population the vast majority of those anomalies are isolated abnormalities without concomitant other congenital heart defects. (Folia Morphol 2015; 74, 1: 73-77)

Key words: coronary vessel anomalies, congenital heart defects, multislice computed tomography

\section{INTRODUCTION}

Until the last decade, the detection of abnormally originating coronary artery was performed using invasive coronary angiography (ICA), sectional examination or echocardiography $[1,2,6,7,10,19,20]$. The recent invention of multidetector cardiac computed tomography (CT) allowed for non-invasive examination of coronary arteries, including their origin [13].

The prevalence of coronary artery anomalies depends on the diagnostic method used for its detec- tion, adopted definition of coronary anomaly and characteristics of the study group [17]. The sectional analysis of the 18,950 consecutive specimens [1] revealed only $54(0.3 \%)$ coronary anomalies, however this study was performed in the middle of the last century when the coronary anomaly was not precisely defined. In the largest population analysis of over 100,000 ICA performed in a period of almost 30 years, the abnormal aortic coronary artery origin occurred in $1.2 \%$ of the evaluated group [19]. In the 
biggest so far echocardiographic study performed on asymptomatic children [6] the anomalous aortic origin of the coronary artery was present in $4(0.2 \%)$ cases. Due to its recent application in the clinical practice, there is only a few data on the incidence of coronary anomalies in CT [16].

Majority of coronary anomalies remain isolated defects [15], but may also present with concomitant other malformations such as transposition of great vessels, tetralogy of Fallot, single ventricle or persistent truncus arteriosus $[5,7,15]$. To our knowledge, there is lack of data regarding occurrence of coronary anomalies and other cardiac defects in CT. Therefore, the aim of the study is to assess the prevalence of anomalous origin of the coronary artery in a population that underwent $\mathrm{CT}$ and to evaluate its coexistence with other congenital heart disease.

\section{MATERIALS AND METHODS}

\section{Study population}

7,115 patients, who underwent 64-slice CT (Somatom Sensation 64 Cardiac, Siemens, Erlangen, Germany) or dual-source CT (Somatom Definition, Siemens, Erlangen, Germany) in the years 2005-2011 due to suspected coronary artery disease or in order to evaluate congenital heart disease were also screened for the presence of anomalous origin of the coronary artery. The exclusion criteria were: prior revascularisation therapy including percutaneous coronary interventions and coronary artery bypass grafting (concerned 1,063 patients); contraindications to perform cardiac CT including respiratory failure, severe heart failure, irregular heart rate, pregnancy, known allergy to iodinated contrast agent, impaired renal function (serum creatinine $\geq 1.5 \mathrm{mg} / \mathrm{dL}$ ) and thyroid disorders [4].

\section{CT acquisition}

The contrast-enhanced, retrospectively electrocardiogram-gated image acquisitions were performed during inspiratory breath hold. The imaging parameters common for 64-slice and dual-source CT were tube voltage of 100-120 kV and effective tube current of 350-400 mA. The collimation and temporal resolution revealed: $2 \times 64 \times 0.6 \mathrm{~mm}$ and $330 \mathrm{~ms}$ for 64-slice CT, $2 \times 32 \times 0.6 \mathrm{~mm}$ and $165 \mathrm{~ms}$ for dual-source CT. Electrocardiographically controlled tube current modulation was applied in the majority of patients. The arrival time of contrast agent to the ascending aorta was determined at the level of the carina with use of the test bolus method. Images were reconstructed with a B26f and B46f kernel and an image matrix of $512 \times 512$ pixels.

\section{$\mathrm{CT}$ analysis}

The post processing and study evaluation was performed using a dedicated workstation (Aquarius, TeraRecon, San Mateo, USA). Patients were assessed for the presence of anomalous origin of coronary artery using axial, curved multiplanar (MPR), maximum intensity projection (MIP) and volume rendered technique (VRT) reconstructions. The analysis was performed in the diastolic phase of the cardiac cycle and the modified American Heart Association 16-segment coronary artery model was utilised [9].

Anomalous origin of the coronary artery was named and classified according to Jacobs [8]. This classification includes: anomalous pulmonary origin of coronary artery (APOC) and anomalous aortic origin of coronary artery (AAOC). AAOC is divided into subtypes: anomalous origin of the coronary artery from the wrong coronary sinus, single coronary artery, multiple ostia (left main coronary artery atresia) and high take-off of the coronary artery. Due to ambiguous criteria and little clinical importance $[12,13]$ patients with multiple ostia and high take-off of the coronary artery were not taken into analysis. All patients were evaluated for the presence of concomitant other heart defects.

\section{RESULTS}

Anomalous origin of coronary artery was found in $62(0.87 \%)$ patients. Among them 34 (54\%) were males and $28(46 \%)$ were females. The mean age of the group of patients with anomalies was $57.4 \pm$ \pm 15.1 years with the minimum and maximum age of 17 and 80 years, respectively.

APOC was present in $3(0.04 \%)$ cases and concerned only patients with left coronary artery (LCA) anomalous pulmonary origin (ALCAPA) (Fig. 1A), mean age $34.3 \pm 10.3$ years. AAOC revealed in $59(0.83 \%)$ subjects and concerned:

$-23(0.32 \%)$ patients with anomalous origin of the circumflex artery from the right coronary artery sinus (ALCX);

- $18(0.25 \%)$ patients with anomalous origin of the right coronary artery from the left coronary artery sinus (ARCA) (Fig. 1B);

- $13(0.18 \%)$ patients with anomalous origin of the left coronary artery from the right coronary artery sinus (ALCA) (Fig. 1C); 


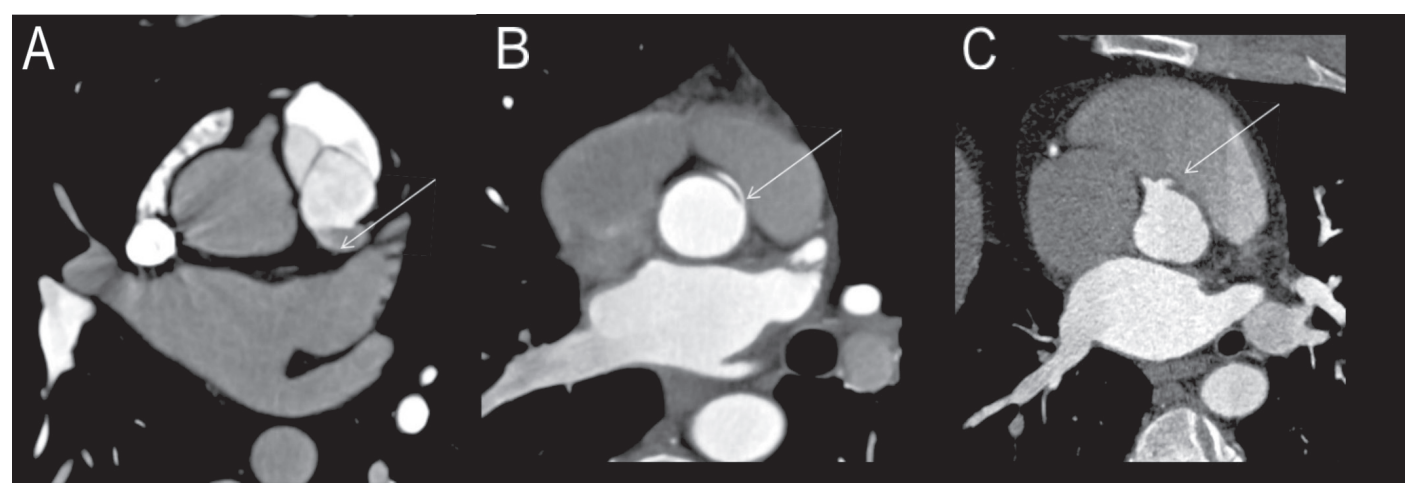

Figure 1. Computed tomography multiplanar reconstruction (MPR) views of the anomalously originating coronary arteries (arrows); A. Anomalous pulmonary origin of the left coronary artery; B. Anomalous origin of the right coronary artery from the left coronary artery sinus; $\mathbf{C}$. Anomalous origin of the left coronary artery from the right coronary artery sinus.

- $3(0.04 \%)$ patients with anomalous left coronary artery originating from the non-coronary artery sinus;

$-2(0.03 \%)$ patients with anomalous origin of the left anterior descending artery from the right coronary artery sinus (ALAD).

Concomitant heart defects were observed in $5(0.07 \%)$ patients (mean age $37.2 \pm 17.8$ years), all with $A A O C$, malformations included:

- $4(0.066 \%)$ patients with transposition of great arteries ( 2 with ALAD, 1 with ALCx and 1 subject with ARCA);

- single ventricle in $1(0.01 \%)$ patient with ARCA and concomitant atrial septal defect (ASD).

Thus 57 (92\%) subjects revealed isolated anomalous coronary artery origin.

Transposition of great arteries (TGA) concerned 2 patients with dextro-transposition (d-TGA) of the great arteries (1 patient with ALCx and 1 subject with ARCA) and 2 subjects with levo-transposition (I-TGA) of the great arteries ( 2 patients with ALAD).

One subject with d-TGA (ARCA) as well as both patients with I-TGA revealed ventricular septal defect (VSD). Patient with d-TGA (ARCA) also revealed ASD (apart from VSD). The second patient with d-TGA (ALCX) presented with concomitant persistent ductus arterious (PDA).

\section{CT data}

CT examination was performed in 8 (17\%) patients using 64-slice scanner and in 54 (83\%) using dual-source device. The mean dose of radiation obtained during the examination revealed $11.2 \pm$ $\pm 3.3 \mathrm{mSv}$ for a 64-slice and $13.5 \pm 3.0 \mathrm{mSv}$ for dual-source device.

\section{DISCUSSION}

The present study demonstrates similar prevalence of the anomalous origin of a coronary artery as in the study of invasive angiograms performed by Yamanaka et al. [19]. Also the average age of the study group was similar in both studies (57 and 53 years for CT and ICA analysis, respectively). In the above cited publication the authors analysed 126,595 angiograms and revealed occurrence of the AAOC in 1686 (1.3\%) cases. However, if applied the same definition and criteria of coronary anomaly as in our study, the prevalence of the anomalous coronary artery origin in a publication by Yamanaka et al. [19] would be $0.58 \%$ of the analysed patients. The comparison of both analyses revealed similar incidence of ALCAPA, ALCx and ALAD, while occurrence of ARCA and ALCA was different in favour of our study. This difference was threefold and tenfold with regard to ARCA and ALCA, respectively. The discrepancy between Yamanaka et al. [19] and our analysis may result from at least two reasons. First of them is different inclusion criteria for both studies, as patients with other congenital heart defect were excluded by Yamanaka et al. [19]. Second, the invasive angiographic study was performed in the second half of the last century when diagnostic coronary imaging was less available than is nowadays.

The study of 1,950 ICA by Angelini et al. [2] revealed higher incidence (5.6\%) of coronary anomalies than our analysis. Such a big discrepancy is a consequence of a wide definition of coronary anomaly introduced by the authors and evaluation of a different group of patients than in our study, as Angelini et al. [2] included subjects with known or suspected coronary artery disease. The echocardiographic study by Davis et al. [6] was performed on adolescent 
asymptomatic subjects, which resulted in a low incidence of coronary anomalies in those patients. This suggestion is consistent with results of an another echocardiography analysis by Zeppilli et al. [20] who performed a study in asymptomatic athletes and revealed only $3(0.09 \%)$ patients with anomalous origin of a coronary artery.

Frescura et al. [7] published an autopsy analysis, which revealed $27(2.2 \%)$ cases of isolated anomalous origin of a coronary artery out of 1,200 evaluated cases with congenital heart disease. However comparison of the anomalies incidence in study by Frescura et al. [7] with our analysis is impossible due to divergence of study groups. A sectional analysis by Alexander et al. [1] revealed only $54(0.3 \%)$ coronary anomalies, but again it is incomparable with other publications as the authors of this study applied different definition of an anomaly.

According to an analysis by Williams et al. [18] an anomalous pulmonary origin of a coronary artery was present in $0.01 \%$ of population. Thus concerns left coronary artery anomalous pulmonary origin (ALCAPA) and 4 times less prevalent right coronary artery anomalous pulmonary origin (ARCAPA), with the incidence of $0.008 \%$ and $0.002 \%$, respectively. In our study we found only 3 patients with ALCAPA (also known as Bland-White-Garland syndrome) with its prevalence of $0.04 \%$. The reason for this discrepancy may be multifactorial. First, our study was performed in a group with higher average age; second, we evaluated patients using CT while Williams et al. [18] used other modalities including ICA and echocardiography. Lastly, patients in our analysis had their CT performed recently, while Williams et al. [18] evaluated cases mostly from the last century, when the diagnostic imaging was much less available.

The results of the recently published analysis [16] of incidence of the coronary anomalies originating from the opposite sinus of Valsalva in CT are consistent with our study, as Opolski et al. [16] revealed coronary anomaly in $0.84 \%$ of patients $(72$ of 8,522$)$. Moreover the study by Opolski et al. [16] was performed in a study group of similar quantity. The main difference between the 2 studies is that the authors of the above cited analysis excluded patients with complex congenital heart disease. Analysis of coexistence of anomalous origin of a coronary artery with other congenital heart disease in CT makes our study innovative.

In our analysis the type of concomitant heart defects coexisting with coronary anomaly remain consistent with other publications concerning this topic $[5,7,11,14,15]$. Kaushal et al. [11] in an analysis of 27 adolescents revealed coexistence of ASD (4 patients) and PDA (1 subject) with anomalous origin of a coronary artery in $5(18 \%)$ cases. In comparison, we discovered a coexistence of other heart defects in $6(8 \%)$ patients. The coincidence appeared to be lower in our study; however, in our population patients presented with a more complex congenital heart disease such as TGA and single ventricle. In this group ASD (1 patient) and PDA (1 patient) coexisted in 2 cases, both with d-TGA. This difference may result from the lower average age in the publication by Kaushal et al. [11] as well as from the fact that Kasushal et al. [11] included in their study only patients scheduled for surgical repair of the AAOC. This implied that the population in this study didn't reveal complex malformations such as TGA. On the other hand, we included and analysed all patients that underwent cardiac $\mathrm{CT}$, also those with complex heart defects. This also enables us to state that we assessed the prevalence of the complex congenital heart defects that included anomalous origin of a coronary artery in a population that underwent cardiac CT.

However, the evaluated patients were selected group with different indications to perform cardiac $\mathrm{CT}$. This results in the biggest limitation of our study as we didn't assess the prevalence of anomalous origin of a coronary artery in the unselected population, but among those scheduled for cardiac CT. Another limitation of our analysis is the high radiation dose obtained by patients in $\mathrm{CT}$, as in attempt to reduce radiation exposure, more $\mathrm{CT}$ studies are now being performed using prospective gating as well as reduced tube voltage [3]. Lastly, the retrospective nature of the analysis is also a limitation of this study.

\section{CONCLUSIONS}

The incidence of anomalous origin of a coronary artery in cardiac CT is similar to studies based on invasive coronary angiography. In an adult population the vast majority of those anomalies are isolated abnormalities without concomitant other congenital heart defects. 


\section{REFERENCES}

1. Alexander RW, Griffith GC (1956) Anomalies of the coronary arteries and their clinical significance. Circulation, 14: 800-805.

2. Angelini P, Velasco JA, Flamm S (2002) Coronary anomalies: incidence, pathophysiology, and clinical relevance. Circulation, 105: 2449-2454.

3. Bischoff B, Hein F, Meyer T, Krebs M, Hadamitzky M, Martinoff S, Schömig A, Hausleiter J (2010) Comparison of sequential and helical scanning for radiation dose and image quality: results of the Prospective Multicenter Study on Radiation Dose Estimates of Cardiac CT Angiography (PROTECTION) I Study. Am J Roentgenol, 194: 1495-1499.

4. Cademartiri F, Casolo G, Midiri M (2008) Clinical aplications of cardiac CT. Springer-Verlag, Milan.

5. Dabizzi RP, Teodori G, Barletta GA, Caprioli G, Baldrighi G, Baldrighi V (1990) Associated coronary and cardiac anomalies in the tetralogy of Fallot. An angiographic study. Eur Heart J, 1: 692-704.

6. Davis JA, Cecchin F, Jones TK, Portman MA (2001) Major coronary artery anomalies in a pediatric population: incidence and clinical importance. J Am Coll Cardiol, 37: 593-597.

7. Frescura C, Basso C, Thiene G, Corrado D, Pennelli T, Angelini A, Daliento L (1998) Anomalous origin of coronary arteries and risk of sudden death: a study based on an autopsy population of congenital heart disease. Hum Pathol, 29: 689-695.

8. Jacobs ML, Mavroudis C (2010) Anomalies of the coronary arteries: nomenclature and classification. Cardiol Young, 20: 15-19.

9. Kaiser C, Bremerich J, Haller S, Brunner-La Rocca HP, Bongartz G, Pfisterer M, Buser P (2005) Limited diagnostic yield of non-invasive coronary angiography by 16 -slice multi-detector spiral computed tomography in routine patients referred for evaluation of coronary artery disease. Eur Heart J, 26: 1987-1992.

10. Kardos A, Babai L, Rudas L, Gaál T, Horváth T, Tálosi L, Tóth $K$, Sárváry L, Szász K (1997) Epidemiology of congenital coronary artery anomalies: a coronary arteriography study on a central European population. Cathet Cardiovasc Diagn, 42: 270-275.

11. Kaushal S, Backer CL, Popescu AR, Walker BL, Russell HM, Koenig PR, Rigsby CK, Mavroudis C (2011) Intramural coronary length correlates with symptoms in patients with anomalous aortic origin of the coronary artery. Ann Thorac Surg, 92: 986-991.

12. Kayalar N, Burkhart HM, Dearani JA, Cetta F, Schaff HV (2009) Congenital coronary anomalies and surgical treatment. Congenit Heart Dis, 4: 239-251.

13. Kim SY, Seo JB, Do KH, Heo JN, Lee JS, Song JW, Choe YH, Kim TH, Yong HS, Choi SI, Song KS, Lim TH (2006) Coronary artery anomalies: classification and ECG-gated multi-detector row CT findings with angiographic correlation. Radiographics, 26: 317-333.

14. Mawson JB (2002) Congenital heart defects and coronary anatomy. Tex Heart Inst J, 29: 279-289.

15. Montaudon M, Latrabe V, Iriart X, Caix P, Laurent F (2007) Congenital coronary arteries anomalies: review of the literature and multidetector computed tomography (MDCT)-appearance. Surg Radiol Anat, 29: 343-355.

16. Opolski MP, Pregowski J, Kruk M, Witkowski A, Kwiecinska S, Lubienska E, Demkow M, Hryniewiecki T, Michalek P, Ruzyllo W, Kepka C (2013) Prevalence and characteristics of coronary anomalies originating from the opposite sinus of Valsalva in 8,522 patients referred for coronary computed tomography angiography. Am J Cardiol, 111: 1361-1367.

17. Warnes CA, Williams RG, BashoreTM, Child JS, Connolly HM, Dearani JA, Del Nido P, Fasules JW, Graham TP Jr, Hijazi ZM, Hunt SA, King ME, Landzberg MJ, Miner PD, Radford MJ, Walsh EP, Webb GD (2008) ACC/AHA 2008 Guidelines for the Management of Adults with Congenital Heart Disease: Executive Summary: a report of the American College of Cardiology/American Heart Association Task Force on Practice Guidelines (writing committee to develop guidelines for the management of adults with congenital heart disease). Circulation, 118: 2395-2451.

18. Williams IA, Gersony WM, Hellenbrand WE (2006) Anomalous right coronary artery arising from the pulmonary artery: a report of 7 cases and a review of the literature. Am Heart J, 152: e9-e17.

19. Yamanaka O, Hobbs RE (1990) Coronary artery anomalies in 126,595 patients undergoing coronary arteriography. Cathet Cardiovasc Diagn, 21: 28-40.

20. Zeppilli P, dello Russo A, Santini C, Palmieri V, Natale L, Giordano A, Frustaci A (1998) In vivo detection of coronary artery anomalies in asymptomatic athletes by echocardiographic screening. Chest, 114: 89-93. 